\title{
Laser induced impact ionization in semiconductors: A Monte Carlo study for silicon
}

\author{
Boaz Kochman, Keesoo Yeom, and Jasprit Singh \\ Department of Electrical Engineering and Computer Science, The University of Michigan, Ann Arbor, \\ Michigan 48109-2122
}

(Received 9 November 1995; accepted for publication 25 January 1996)

\begin{abstract}
Monte Carlo computer simulations are carried out to study impact ionization due to a sinusoidal field present in high-power laser pulses. As an application we study the impact ionization coefficient, $\alpha$, for electrons in silicon as a function of the field frequency, pulse width, and the rms value of the field. In all cases we stay below the frequency values where band-to-band absorption would create electron-hole pairs. As is the case for constant (dc) fields, $\log \alpha$ is found to be linear with field strength. For fields oscillating at frequencies much below the inverse of the carrier scattering rate, the impact ionization coefficient is found to have the same value as in the constant field case with the rms field replacing the dc value. At higher frequencies the impact ionization rate decreases. The dependence of $\alpha$ on field frequency and pulse width is studied. (C) 1996 American Institute of Physics. [S0003-6951(96)00814-3]
\end{abstract}

Impact ionization related breakdown of semiconductors plays a very important role in microelectronics. This phenomenon limits the high-power performance of transistors since at high applied biases, the current in the device increases uncontrollably due to carrier multiplication. This phenomenon is also exploited for the design of avalanche photodetectors to provide high gain. Knowledge of the impact ionization coefficient is, therefore, of great importance. The experimental measurement of the impact ionization coefficient is rather difficult due to the difficulty of maintaining uniform fields and avoiding current instabilities. As a result, there is considerable uncertainty in the impact ionization coefficients ( $\alpha$ for electrons, $\beta$ for holes) even in widely used semiconductors such as $\mathrm{Si}, \mathrm{GaAs}$, and $\mathrm{InP}$. One technique that has recently been successfully applied to this problem is the use of short-pulse, high-power lasers to create impact ionization. ${ }^{1-3}$ In these pump-pulse experiments a high-power short-pulse laser with photon energies smaller than the band gap impinges upon the sample. Impact ionization results in plasma formation which is detected by a low-power probe beam. As long as the photon energy is smaller than the band gap, the dominant carrier generation process is through impact ionization. The use of short-pulse width, high-power laser pulses to create impact ionization can also be exploited for many interesting devices. In order to exploit the potential of this technique, it is important to develop an understanding of how short-laser pulses cause breakdown in semiconductors. In this letter, we carry out a Monte Carlo study of impact ionization caused by a high-frequency electric field (present in a laser pulse) in silicon. The study examines the dependence of impact ionization coefficient for electrons on the frequency, field strength, and the duration of the optical pulse.

The code used for the Monte Carlo based computer simulation is based on a code previously used to study the response of electrons to a dc electric field. ${ }^{4}$ It has been extended to include sinusoidal fields. To describe the band structure of electrons we use a nonparabolic ellipsoidal conduction band:

$$
E(1+\alpha E)=\hbar^{2}\left(\frac{k_{x}^{2}+k_{y}^{2}}{2 m_{t}^{*}}+\frac{k_{z}^{2}}{2 m_{l}^{*}}\right) .
$$

Both $X$ and $L$ valleys are included in the simulations. The dominant scattering mechanisms are the $g$ - and $f$-optical phonon scattering (emission and absorption) and acoustic scattering.

To study the problem of breakdown in a laser field, we use two models for the impact ionization rate in silicon. The first model is a "super-soft", model from Thoma et al.," where the rate goes as the cube of $\left(E-E_{\text {th }}\right)$ near threshold rather than the square. The impact ionization rate is given by

$$
\begin{aligned}
& R_{\text {impact }} \\
& = \begin{cases}0.875 \times 10^{13}\left(\frac{E}{\mathrm{eV}}-1.128\right) \mathrm{s}^{-1}, & 1.128<\frac{E}{\mathrm{eV}}<1.750 \\
6.646 \times 10^{13}\left(\frac{E}{\mathrm{eV}}-1.572\right) \mathrm{s}^{-1}, & 1.750<\frac{E}{\mathrm{eV}} .\end{cases}
\end{aligned}
$$

The second model is a Keldysh-like formula which was derived by Cartier et al. by fitting Monte Carlo results to experimental data. ${ }^{6}$ The impact ionization rate is given by

$$
R_{\text {impact }}=\sum_{i=1}^{3} \theta\left(E-E_{\mathrm{th}}^{(i)}\right) P^{(i)}\left(\frac{E-E_{\mathrm{th}}^{(i)}}{E_{\mathrm{th}}^{(i)}}\right)^{2},
$$

where $E_{\mathrm{th}}^{(i)}=1.2,1.8$, and $3.45 \mathrm{eV}, P^{(i)}=6.25 \times 10^{10}, 3.0$ $\times 10^{12}$, and $6.8 \times 10^{14} \mathrm{~s}^{-1}$, for $i=1,2$, and 3 , respectively, and $\theta$ is the step function.

The models gave the same form of results for the impact ionization coefficient both as a function of field strength and field frequency. Thus the general results are independent of the particular model used for impact ionization rate. We compared the impact ionization coefficient for a dc field with experimental data. Cartier's model gives good agreement with experimental results. Thoma's model, on the other hand, has been known to yield values that are too high, and, indeed, that is what we have found. 


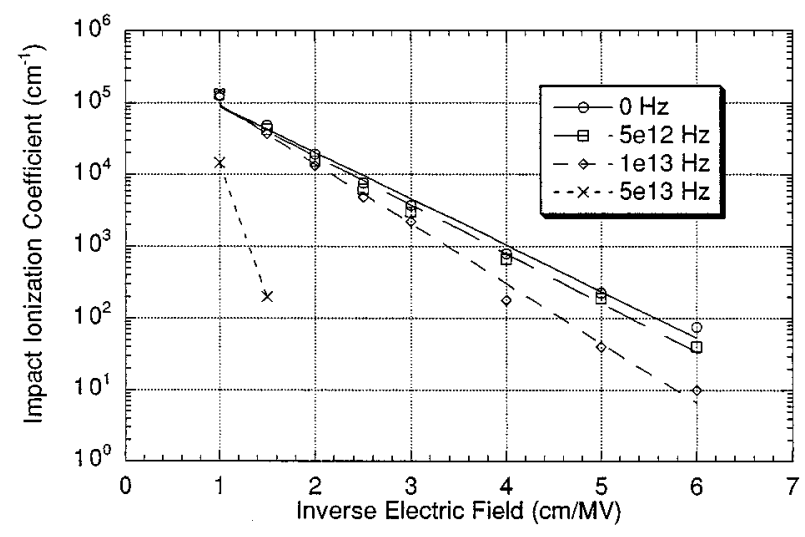

FIG. 1. Field strength dependence of the impact ionization parameter for 10 ps pulse with a constant and several sinusoidal fields. In the ac cases, the given field values are rms.

To extend the study to include the response of the electrons to short-pulse, high-power lasers, we use the following equation to represent the intercollisional free flight

$$
\frac{d \mathbf{p}}{d t}=q \mathbf{E}_{0} \sin (\omega \mathbf{t})
$$

Equation (3) is then integrated to give the change in momentum during the free flight

$$
\mathbf{p}(t+\Delta t)-\mathbf{p}(t)=\frac{q \mathbf{E}_{0}}{\omega}\{\cos (\omega t)-\cos [\omega(t+\Delta t)]\},
$$

what $t$ is the time before the free flight and $\Delta t$ is the length of the free flight.

In Fig. 1 we show the impact ionization coefficient calculated using the Cartier model for a 10 ps pulse with different frequencies. The dc results show good agreement with experiment. The Thoma model is not included in the plot since the form of the results is the same while the actual values are too high. We note that once the frequency reaches a low value $\left(5 \times 10^{12} \mathrm{~s}^{-1}\right)$, the impact ionization coefficient approaches the dc value. Note that in Fig. 1, the field values for the ac case are the rms values. As the frequency increases, the value of $\alpha$ in the ac case becomes smaller than that of the dc case. We also note that the discrepancy between the ac and the dc $\alpha$ values grows at lower fields.

To understand the dependence of $\alpha$ on frequency and the rms field, it is important to understand the role scattering plays in the impact ionization process. As can be seen from Eqs. (2) and (3), for impact ionization to occur, the initial electron's energy must exceed the threshold energy. In absence of scattering, in an ac field the electron energy oscillates and the mean energy is simply given by

$$
\langle E\rangle=\frac{e^{2} E_{0}^{2}}{4 m^{*} \omega^{2}},
$$

where $m^{*}$ is the mass along the polarization direction.

In the presence of scattering, the electron does not follow the field and can gain energies much larger than that given by the equation above. To illustrate this, in Fig. 2 we show the average energy of an ensemble of electrons as an optical pulse impinges on the semiconductor. For the parameters shown in Fig. 2 we see that the electrons start to gain

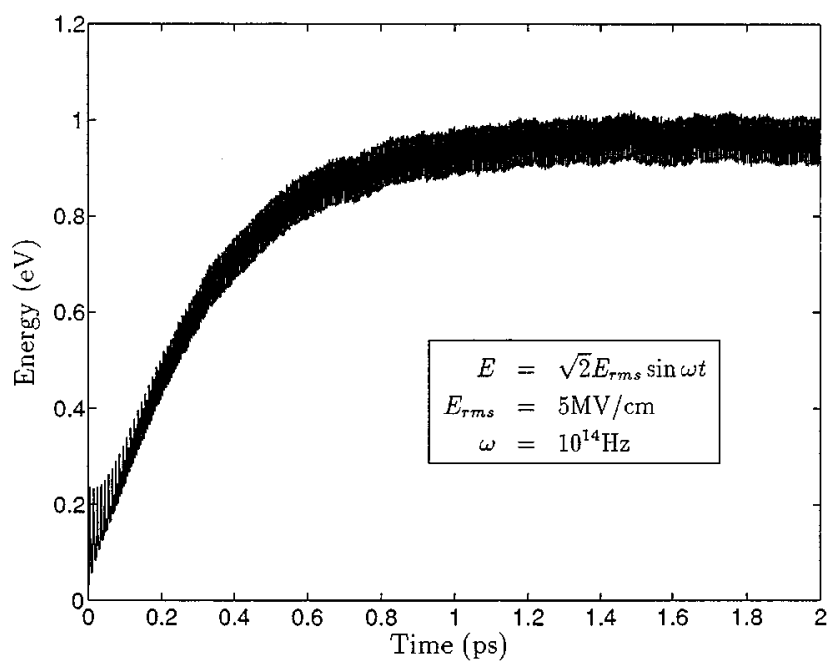

FIG. 2. Average electron energy as a function of time for a sinusoidal field. The rms field is $5 \mathrm{MV} / \mathrm{cm}$. The frequency is $1 \times 10^{14} \mathrm{~Hz}$.

energy from the field and reach a steady-state value within one picosecond. In the case depicted in Fig. 2, the average energy under steady-state conditions is $0.82 \mathrm{eV}$ with scattering. If the scattering is turned off in the simulation, the average energy is only $0.11 \mathrm{eV}$ consistent with the value given by Eq. (6). It may be pointed out that if a high density plasma is produced by the laser pulse, carrier-carrier scattering would also be important randomizing the energy of the electron gas. As a result the initial oscillations shown in Fig. 2 may not be present.

The results of Fig. 2 can be used to understand the dependence of $\alpha$ on frequency and field. At very high frequencies, the electron can follow the field for several cycles without scattering so that the average energy of the electrons is smaller than the value where the electron suffers several scattering events within each cycle. Also, as the field is increased, the overall scattering rate increases mainly because of higher density of states for higher energy electrons. Thus a frequency that is high enough to avoid scattering in a given cycle at low fields may not be high enough at high fields. As a result, as the field is increased, the difference between the ac and dc $\alpha$ values becomes smaller.

We have also studied the effect of field frequency on impact ionization, with results for both the Cartier model and the Thoma model given in Fig. 3. Both of the models give the same form for the results, but the Thoma model yields higher values than the Cartier model. At dc, experimental results agree with the Cartier model. In $\mathrm{SiO}_{2}$, it has been found experimentally that the impact ionization coefficient produced by a dc field is approximately equal to that produced with a laser pulse of the same strength, so long as the rms value is used. We have found this to be true in silicon for field frequencies below $10^{13} \mathrm{~Hz}$. In this range the field frequency is much lower than the typical scattering rate, so this result seems reasonable. In the $10^{13}$ to $5 \times 10^{14} \mathrm{~Hz}$ range, the impact ionization coefficient and the average energy decrease dramatically. This is the range where the field frequency is comparable to the typical scattering rate. Above $5 \times 10^{14}$ the field frequency is much greater than the scattering rate, the average energy levels off at a low value, and no 


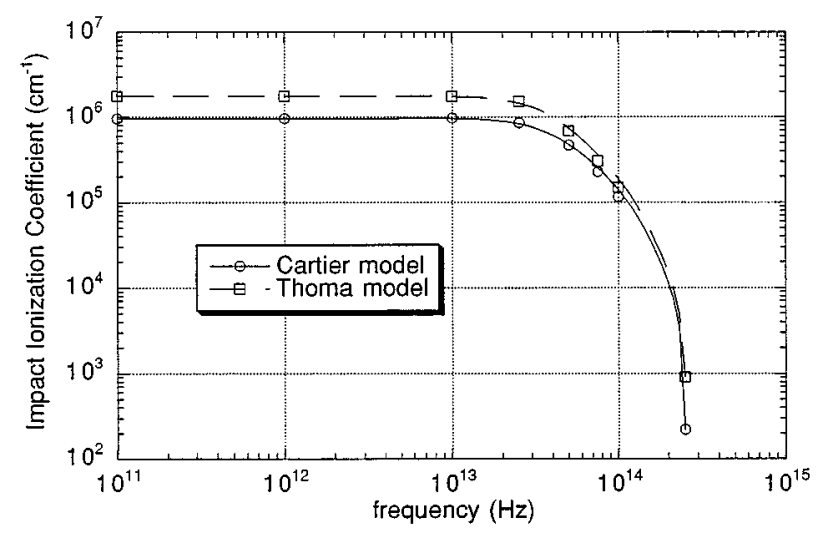

FIG. 3. Frequency dependence of the impact ionization parameter for a 10 ps pulse with an rms field strength of $3 \mathrm{MV} / \mathrm{cm}$.

impact ionization occurs. As might be expected, at these very high frequencies, the scattering plays little role since it cannot keep up with the field. Running the simulations with scattering turned off yields the same results.

It has been suggested that the rms breakdown field corresponds to the dc breakdown field, at least in ionic solids, by the relationship ${ }^{3}$

$$
E_{\mathrm{th}}^{\mathrm{rms}}(\omega)=E_{\mathrm{th}}^{\mathrm{dc}}\left(1+\omega^{2} \tau^{2}\right)^{1 / 2},
$$

where $\tau$ is the collision time. There is some ambiguity in $\tau$ since collision time is a dependent on field strength. We used the collision time at the ac breakdown field, as calculated by Monte Carlo. We found that the ac breakdown field as calculated by Eq. (6) does not agree with breakdown field as determined by the ac Monte Carlo. Thus for silicon the relation given by Eq. (6) does not appear to hold and cannot be used to infer the dc breakdown from the ac breakdown.

In summary, we have carried out Monte Carlo based simulations for impact ionization breakdown in silicon. The dependence of the breakdown frequency and field strength have been explored. The overall form of the results doe not depend on the particular model used for impact ionization rate.

This work was funded by the U.S. Army URI program (DAAL03-92-G-0109) and a grant from the U.S. Air Force (Grant No. AFOSR-91-0349).

${ }^{1}$ D. Du, X. Liu, G. Korn, J. Squier, and G. Mourou, Appl. Phys. Lett. 64, 3071 (1994)

${ }^{2}$ E. Yablonovitch and N. Bloembergen, Phys. Rev. Lett. 29, 907 (1972).

${ }^{3}$ N. Bloembergen, IEEE J. Quantum Electron. QE-10, 375 (1974).

${ }^{4}$ K. Yeom, J. Hinckley, and J. Singh, Appl. Phys. Lett. 64, 2985 (1995).

${ }^{5}$ R. Thoma, H. J. Peifer, and W. L. Engl, J. Appl. Phys. 69, 2300 (1991).

${ }^{6}$ E. Cartier, M. Fischetti, E. Eklund, and F. McFeely, Appl. Phys. Lett. 62, 3339 (1993). 